\title{
Letters
}

\section{Novel drug of choice in Eaton-Lambert syndrome}

Sir: Muscle weakness in the myasthenic syndrome of Eaton-Lambert' is caused by diminished transmitter release from motor nerve endings ${ }^{2}$ secondary to morphological destruction of the active release zones in the nerve terminals ${ }^{3}$ which is thought to have an autoimmune aetiology. ${ }^{45} \mathrm{Im}$ munosuppressive therapy has recently been recommended ${ }^{5}$ but its full effect is delayed by several months and severe side effects may be expected during prolonged treatment. Symptomatic treatment with an immediately acting drug would be of value to the cancer patient with Eaton-Lambert syndrome, and if such a drug is proved safe it could also be used in the cryptogenetic form of the disease. 4-aminopyridine has been tested, ${ }^{6-8}$ but its usefulness is limited by central nervous system stimulant effects sometimes causing seizures. We have tried another aminopyridine, 3,4-diaminopyridine $(3,4-D A P)$, in three patients with Eaton-Lambert syndrome. This drug is known from animal experiments to be more potent in improving neuromuscular transmission 9 and less convulsant ${ }^{10}$ than 4-aminopyridine.

Our first patient was a 70-year-old woman with cryptogenic Eaton-Lambert syndrome with marked difficulties in walking, pronounced dysarthria, ptosis, difficulty in swallowing, and constipation and inability to urinate. She further deteriorated and required mechanical respiratory assistance because of respiratory muscle weakness. The intravenous injection through a central venous cannula of $8 \mathrm{mg} \mathrm{3,4-DAP}$ within 5 minutes caused marked improvement. The patient was able to breathe without respiratory assistance, ptosis disappeared, facial expression reappeared and muscle strength in arms and legs improved a lot. Examination of neuromuscular transmission by repetitive nerve stimulation showed marked decrease of the neuromuscular block. The i.v. injection caused much salivation and secretion from the respiratory tract, which was blocked by atropine intravenously. Treatment with repeated intravenous infusions of 3,4-DAP gave satisfactory clinical and electrophysiological improvement (fig). She received daily intravenous infusions of 6-9 mg 3,4-DAP 4 times a day with $0.5 \mathrm{mg}$ atropine for 5 months without side effects. During this treatment she could breathe

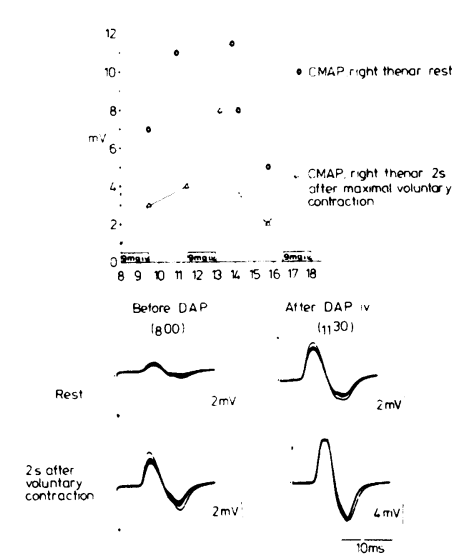

Fig Augmentation of the compound muscle action potential (CMAP) of the right thenar muscles at supramaximal stimulation of the median nerve after intravenous infusion of 3,4-diaminopyridine (DAP). The CMAPs at rest and $2 s$ after maximal voluntary contraction are illustrated and plotted in the diagram. Note different amplification of the lower right record.

without a respirator, walk with a stick, swallow normally and her urethral catheter could be removed. Oral administration of 3,4-DAP was also effective and later she was treated by $24 \mathrm{mg}$ orally 4 times a day with equally good results. Interestingly, previous attempt to treat this patient with 4-aminopyridine had caused a fit.

Our second patient also had cryptogenetic Eaton-Lambert syndrome. A 72-yearold woman with proximal muscle weakness and slight cranial nerve muscle symptoms clinically and electrophysiologically deteriorated in 1982 . Her ability to walk decreased from 1000 to $200 \mathrm{~m}$ and she could no longer climb the stairs to her apartment. Intravenous injection of $8 \mathrm{mg}$, 3,4-DAP and oral administration of $30 \mathrm{mg}$ markedly improved all muscle functions tested. Electrophysiological examination demonstrated maximum effect $1 \frac{1 / 2}{h}$ after an oral dose and disappearance of effect about $2 \mathrm{~h}$ later. Continuous oral treatment with $18 \mathrm{mg} 4$ times a day drastically changed daily life of this patient from being a cripple to that of an almost normal retired lady being able to walk more than $1000 \mathrm{~m}$ and climb stairs. She has now been treated with this dose for three months without side effects except temporary perioral paraesthesias and transitory pain in her arm after i.v. injection.

Our third patient with Eaton-Lambert syndrome was a 62-year-old woman with cancer of the gallbladder with metastasis to the liver. She had marked proximal muscle weakness with inability to walk, dysarthria and dysphagia. An i.v. infusion of $8 \mathrm{mg}$ 3,4-DAP or $24 \mathrm{mg}$ orally markedly improved muscle function and she could walk with minor support and her. speech improved. Treatment with $24 \mathrm{mg}$ 3,4-DAP orally 4 times a day was effective but after a short time interrupted by her death caused by pulmonary embolism.

From our results we.conclude that 3,4DAP is a highly potent and probably safe drug in Eaton-Lambert syndrome. Althouth only symptomatic treatment we suggest it is the present drug of choice in Eaton-Lambert syndrome associated with malignancy. Until the aetiology of the disease has been established and a safe longterm treatment delineated, 3,4-DAP may also be considered the first drug in the more rare cryptogenic form of the disease.

H LUNDH O NILSSON

I ROSEN

Departments of Neurology and Clinical Neurophysiology, University Hospital, S-281 85 Lund,

\section{References}

' Eaton LM, Lambert EH. Electromyography and electric stimulation of nerves in diseases of motor unit: observations on myasthenic syndrome associated with malignant tumours. JAMA 1957;163:1117-24.

${ }^{2}$ Elmqvist D, Lambert EH. Detailed analysis of neuromuscular transmission in a patient with the myasthenic syndrome sometimes associated with bronchogenic carcinoma. Mayo Clin Proc 1968;43:689-713.

${ }^{3}$ Fukunaga H, Engel AG, Osame M, Lambert EH. Paucity and disorganization of presynaptic membrane active zones in the Lambert-Eaton myasthenic syndrome. Muscle Nerve 1982;5:686-97.

4 Gutman L, Crosby TW, Takamori M, Martin JD. The Eaton-Lambert syndrome and autoimmune disorders. Am J Med 1972;53: 354-6.

${ }^{5}$ Lang B, Newsom-Davis J, Wray D, Vincent A. Autoimmune aetiology for myasthenic (Eaton-Lambert) syndrome. Lancet $1981 ; 2: 224-6$.

${ }^{6}$ Lundh H, Nilsson O, Rosen I. 4aminopyridine: a new drug tested in the treatment of Eaton-Lambert syndrome. $J$ Neurol Neurosurg Psychiatry 1977; 40:1109-12.

${ }^{7}$ Murray NMF, Newsome-Davis J. Treatment with oral 4-aminopyridine in disorders of 
neuromuscular transmission. Neurology (NY) 1981;31:265-71.

- Lundh H. Therapeutic applications of aminopyridines in diseases of neuromuscular transmission. In: Lechat $P$, Thesleff $S$, Bowman WC, eds. Aminopyridines and Similarly Acting Drugs. Advances in the Biosciences Vol 35. Oxford: Pergamon Press, 1982:287-96.

' Molgo J, Lundh H, Thesleff S. Potency of 3,4diaminopyridine and 4-aminopyridine on mammalian neuromuscular transmission and the effect of pH changes. Europ J Pharmacol 1980;61:25-34.

${ }^{10}$ Lechat P, Deysson G, Lemeignan M, Adolphe M. Toxicité aigue composeé de quelques aminopyridines in vivo (Souris) et in vitro (cultures cellulaires). Ann Pharmac Fr 1968;26:345-49.

Accepted 10 February 1983

\section{Sustained levodopa therapy in tardive dyskinesia}

Sir: The pathophysiology of tardive dyskinesias is still poorly understood, but striatal post-synaptic dopamine receptor hypersensitivity may be implicated.' ${ }^{\prime}$ Permanent discontinuation of the offending neuroleptic offers the best hope of relief, but the patients' mental state often precludes this. Tetrabenazine is the most effective drug treatment, but side-effects including sedation, depression, Parkinson's syndrome and akathisia are common. The longterm administration of levodopa to rodents has recently been shown to attenuate the behavioural and biochemical features of dopaminergic hypersensitivity. ${ }^{23}$ Promising results have also been reported in tardive dyskinesia giving sustained levodopa treatment ${ }^{2}$ or small doses of dopamine receptor agonists. ${ }^{4}$ In view of these findings we have been encouraged to extend this approach to the treatment of patients with irreversible, persistent dyskinesias no longer receiving neurolpetics.

Seven patients (six female, one male) with moderate or severe tardive dyskinesia agreed to participate. Their mean age was 69 years (range 53-93) and the mean duration of involuntary movements was 7 years (range 3-12). Neuroleptics had been given for a mean period of 9.2 years (range 3-30) for schizophrenia, save for three with chronic dyspepsia, agoraphobia and depression respectively. Their conditions were static and their movement disorders comprised a bucco-linguo-masticatory syndrome in seven, additional limb chorea in five and torticollis in one. Two had coexistent akathisia, but none had Parkin- son's disease. With one exception all had discontinued neuroleptics for at least one year (mean 4 yr) before the trial. Baseline clinical assessments were made by two independent observers using the AIM scale $^{\mathbf{s}}$ and dyskinesia was -recorded simultaneously on video tape. Levodopa $300 \mathrm{mg}$ daily in combination with benserazide was then gradually introduced (Madopar 125, 1 capsule 8 hourly) and the patients assessed at 14 day intervals by the same observers. After a minimum of 12 weeks sustained therapy, patients were re-filmed and the levodopa then discontinued abruptly. Follow-up observation continued for six months with AIM scale scoring.

An initial aggravation of the dyskinesias was seen in one patient following levodopa introduction, but otherwise no significant changes in dyskinesia severity occurred at any stage of the trial.

These disappointing results do not compare favourably with those obtained by Bjørndal and colleagues who reported modest improvement in drug-free patients following one month's levodopa therapy. ${ }^{6}$ Benefit has also been claimed with chronic levodopa in patients still receiving neuroleptics ${ }^{7}$ or in those who have just stopped them. ${ }^{8}$ Casey et al,${ }^{9}$ however, using very large doses of levodopa in combination with benserozide for treatment periods of 8 weeks failed to produce benefit in five neuroleptic treated schizophronics with tardive dyskinesia. In contrast to other studies we also failed to demonstrate an initial increase in dyskinesia following levodopa introduction.? Further studies using dopamine receptor agonists and large doses of levodopa in this refractory group of incapacities are now under way.

RJ HARDIE

AJ LEES

GM STERN

Department of Neurology, University College Hospital, London WC1 6AU, UK

\section{References}

' Marsden CD, Jenner P. The pathophysiology of extrapyramidal side-effects of neuroleptic drugs. Psychol Med 1980;10:55-72.

${ }^{2}$ Alpert M, Friedhoff AJ. Receptor sensitivity modification in the treatment of tardive dyskinesia. Clin Pharmacol Ther 1976;19:103.

${ }^{3}$ Ezrin-Waters C, Seaman P. L-dopa reversal of hyperdopaminergic behaviour. Life Sci 1978;22:1027-32.

4 Carroll BJ, Curtis GC, Kokmen E. Paradoxical response to dopamine agonists in tardive dyskinesia. Am J Psychiat 1977;134;785-9.

s Abnormal involuntary movement scale. In: Guy W, ed. ECDEU Assessment Manual.
Rockville: US Department of Health Education and Welfare. 1976;534-7.

- Bjørndal N, Casey D, Gerlach J. In: Cattabeni F. et al eds, Longterm effects of Neuroleptics. Adv Biochem Psychopharm Vol. 24. New York: Raven Press, 1980;541-5.

' Alpert M, Friedhoff AJ, Diamond F. Use of dopamine receptor agonists to reduce dopamine receptor number in tardive dyskinesia. In: Fahn S, Calne DB, Shoulson I eds Experimental Therapeutics of Movements Disorders. New York: Raven Press, 1983:253-8.

- Shoulson I. Carbidopa/levodopa therapy of co-existent drug-induced Parkinsonism and tardive dyskinesia. In: Fahn S, Calne DB, Shoulson I eds Experimental Therapeutics of Movement Disorders. New York: Raven Press, 1983:259-66.

- Casey DE, Gerlach J, Bjørndal N. Levodopa and receptor sensitivity modification in tardine dyskinesia Psychopharmacology 1982;78:89-92.

\section{Giant aneurysm of the petrous portion of} the carotid artery.

Sir: Aneurysms of the internal carotid artery extracranially are rare; those arising from the petrous segment are particulary unusual. A survey of the literature showed approximately 30 reported cases. ${ }^{1-19}$

A 28-year-old woman had been complaining of severe left ear pain, tinnitus and hearing loss for approximately six months. The pain was continuous and irradiated towards the face, the retromastoid region and sometimes down to her neck. At ENT consultation a vascular mass was found in the left middle ear. An attempted biopsy resulted in massive bleeding controlled by packing the ear. She was then referred to the neurosurgical department with the diagnosis of glomus tumour. Besides a left sensorineural deafness, neurological examination was normal. Both carotid pulsates normally and no bruit was heard about the head and neck. Skull radiographs demonstrated a large area of bone erosion in the left petrous pyramid extending anteriorly into the sphenoid sinus. A sclerotic ring could be noticed around the lytic lesion, suggesting a chronic process. Left carotid arteriography revealed a large $(1.5 \times 2.5 \times 5 \mathrm{~cm}$.) aneurysm located in the petrous segment of the carotid artery projecting laterally into the middle ear, with normal intracranial arborisation. Right carotid arteriography with left carotid compression showed excellent cross filling of the left anterior cerebal and middle cerebral arteries, without aneurysm opacification. 